\title{
Efficacy and safety of ipilimumab in patients with advanced melanoma and brain metastases
}

\author{
Paola Queirolo • Francesco Spagnolo • Paolo Antonio Ascierto - Ester Simeone • \\ Paolo Marchetti - Alessandro Scoppola • Michele Del Vecchio • Lorenza Di Guardo • \\ Michele Maio • Anna Maria Di Giacomo • Andrea Antonuzzo • Francesco Cognetti • \\ Virginia Ferraresi - Laura Ridolfi • Massimo Guidoboni • Michele Guida • \\ Jacopo Pigozzo $\cdot$ Vanna Chiarion Sileni
}

Received: 3 October 2013/Accepted: 31 January 2014/Published online: 16 February 2014

(C) Springer Science+Business Media New York 2014

\begin{abstract}
Patients with melanoma brain metastases have a poor prognosis and historically have been excluded from clinical trials. The Expanded Access Program (EAP) provided an opportunity to evaluate the feasibility of ipilimumab ( $3 \mathrm{mg} / \mathrm{kg}$ every 3 weeks for four doses) in patients with stage 3 (unresectable) or 4 melanoma and asymptomatic brain metastases, who had failed or did not tolerate previous treatments and had no other therapeutic option available. Tumor assessments were conducted at baseline and week 12 using immune-related response criteria and patients were monitored for adverse events (AEs). Of 855 patients participating in the EAP in Italy, 146 had
\end{abstract}

P. Queirolo ( $\square)$

Medical Oncology, IRCCS Azienda Ospedaliera Universitaria

San Martino - IST Istituto Nazionale per la Ricerca sul Cancro, Largo Rosanna Benzi, 10, 16132 Genova, Italy

e-mail: paola.queirolo@hsanmartino.it

F. Spagnolo

Department of Plastic and Reconstructive Surgery - IRCCS San Martino - IST Istituto Nazionale per la Ricerca sul Cancro,

Genova, Italy

\section{P. A. Ascierto - E. Simeone}

Cancer Immunotherapy and Innovative Therapy Unit, National

Cancer Institute IRCCS “Fondazione G. Pascale", Naples, Italy

P. Marchetti · A. Scoppola

Medical Oncology, Dermopathic Institute of the Immaculate IDIIRCCS, Rome, Italy

P. Marchetti

Medical Oncology, Sant' Andrea Hospital, Sapienza University of Rome, Rome, Italy

M. Del Vecchio - L. Di Guardo

Department of Medical Oncology, IRCCS Foundation National

Cancer Institute, Milan, Italy asymptomatic brain metastases. With a median follow-up of 4 months, the global disease control rate was $27 \%$, including 4 patients with a complete response and 13 with a partial response. Median progression-free survival and overall survival were 2.8 and 4.3 months, respectively and approximately one-fifth of patients were alive 1 year after starting ipilimumab. In total, $29 \%$ of patients reported a treatment-related $\mathrm{AE}$ of any grade, which were grade $3 / 4$ in $6 \%$ of patients. AEs were generally reversible with treatment as per protocol-specific guidelines. Ipilimumab shows durable benefits in some patients with advanced melanoma metastatic to the brain, with safety results consistent with those previously reported in clinical trials.

M. Maio - A. M. Di Giacomo

Department of Medical Oncology and Immunotherapy, Tuscan Tumor Institute (ITT), University Hospital of Siena, Siena, Italy

A. Antonuzzo

Department of Medical Oncology, University Hospital Pisa,

"Gathered Hospitals of Santa Chiara", Pisa, Italy

F. Cognetti · V. Ferraresi

Department of Medical Oncology, Regina Elena National Cancer Institute, Rome, Italy

L. Ridolfi · M. Guidoboni

Department of Medical Oncology, Scientific Institute of Romagna for the Study and Treatment of Cancer (IRCCS-IRST), Meldola, Italy

M. Guida

"Giovanni Paolo II" Cancer Institute (IRCCS), Bari, Italy

J. Pigozzo · V. Chiarion Sileni

Melanoma Cancer Unit, Veneto Institute of Oncology IOV-

IRCCS, Padua, Italy 
Keywords Melanoma - Ipilimumab - Expanded access . Brain metastases $\cdot$ Treatment outcome $\cdot$ Safety

\section{Introduction}

Melanoma is the third most common underlying diagnosis in patients with cerebral metastases, after lung and breast cancer $[1,2]$. Indeed, clinically apparent brain metastases are observed in 18-46\% of patients with stage 4 melanoma [3]. Moreover, in our experience, brain metastases are often the first and only site of recurrence in responsive and long-surviving patients with metastatic melanoma.

Few treatment options are available for patients with melanoma brain metastases. Current management typically involves surgery or radiotherapy, with treatment decisions influenced by the number and size of lesions, as well as the extent of extracerebral metastatic disease [4]. A single, or few, accessible lesions might be treatable by surgical excision or stereotactic radiosurgery, with whole brain radiotherapy also offered as a means of providing palliative symptom relief. For patients who refuse radiotherapy, or those who are not eligible for other treatments because they have symptomatic brain metastases or a poor performance status (PS), corticosteroids or other supportive measures may be considered $[1,4,5]$. Chemotherapies can also be used to control the symptoms of brain metastases, but have limited efficacy $[1,2]$. This may reflect the fact that most agents poorly penetrate the blood brain barrier (BBB) and do not cross into the central nervous system (CNS) in adequate quantities to have an antitumor effect [2, 6]. However, there is much debate about the role of the BBB in patients with brain metastases. While some evidence suggests that it is disrupted in patients with established brain metastases, other studies have shown it can be preserved [7, 8].

New treatments are therefore required to improve the prognosis and quality of life of patients with melanoma brain metastases. As patients with brain metastases have been historically excluded from clinical trials due to their poor prognosis, or are often under-represented, limited data are available to support the use of novel treatments in this patient population. There is evidence to suggest that inhibitors of mutated $\mathrm{BRAF}^{\mathrm{V} 600}$ kinase may be an effective treatment option. For example, treatment with vemurafenib, indicated for patients with advanced $\mathrm{BRAF}^{\mathrm{V} 600}$ mutationpositive melanoma, resulted in a disease control rate (DCR) of $67 \%$ in 24 pretreated patients with symptomatic brain metastases [9]. Intracranial objective response rates of 35 and $15 \%$ were reported in pretreated patients with brain metastases and $\mathrm{BRAF}^{\mathrm{V} 600 \mathrm{E}}$ and $\mathrm{BRAF}^{\mathrm{V} 600 \mathrm{~K}}$ mutation-positive melanoma, respectively, following treatment with the BRAF inhibitor dabrafenib [10].
The immunotherapeutic agent ipilimumab is another potential treatment for patients with melanoma brain metastases. By blocking cytotoxic T-lymphocyte associated antigen-4 (CTLA-4), a negative regulator of T-cell activation, ipilimumab potentiates prolonged $\mathrm{T}$-cell activation, proliferation and infiltration into tumors, leading to tumor cell death [11]. Ipilimumab $3 \mathrm{mg} / \mathrm{kg}$ is registered in the European Union for the treatment of adult patients with advanced (unresectable or metastastic) melanoma [12]. In randomized, controlled phase 3 trials, ipilimumab significantly improved overall survival (OS) in both pretreated and treatment-naïve patients with metastatic melanoma $[13,14]$. Many patients treated with ipilimumab in phase 2 and 3 trials, at different doses and combinations, have achieved long-term survival, with 5-year survival rates ranging from 13 to $36 \%[15,16]$. Ipilimumab is generally well tolerated [13], with most adverse events (AEs) manageable using guidelines that have been established throughout the ipilimumab clinical development program $[12,17,18]$.

Clinical trial data suggest that ipilimumab, at a dose of 3 or $10 \mathrm{mg} / \mathrm{kg}$, has activity in patients with brain metastases [13, 19-23]. In a phase 2 prospective trial of patients who were either neurologically asymptomatic $(n=51)$ or symptomatic and on a stable dose of corticosteroids $(n=21)$, the DCR with ipilimumab $10 \mathrm{mg} / \mathrm{kg}$ was 25 and $10 \%$, respectively. Median OS was 7 months for asymptomatic patients, with 1 and 2-year survival rates of 31 and $26 \%$. Equivalent results for patients with symptomatic brain metastases were 3.4 months, with survival rates of 19 and $10 \%$ [19]. In another phase 2 trial investigating the efficacy of ipilimumab $10 \mathrm{mg} / \mathrm{kg}$ plus fotemustine, a chemotherapy known to cross the BBB, 10 out of 20 patients with asymptomatic brain metastases at baseline achieved disease control, including five patients with brain metastases that became undetectable by scan [20]. Based on the results of this trial, a phase 3 trial exploring fotemustine with or without ipilimumab in patients with advanced melanoma and brain metastases is underway.

The activity of ipilimumab in patients with brain metastases is explained by its mechanism of action, as ipilimumab activates the immune system rather than acting on the tumor directly [14]. Current understanding is that T-cells activated by ipilimumab can migrate across the BBB unhindered to mount an intracerebral antitumor immune response [24, 25].

Here, findings are reported from Italian centers participating in an Expanded Access Program (EAP) which included a subset of patients with brain metastases [26]. The EAP provided an opportunity to treat patients with ipilimumab at its licensed dose of $3 \mathrm{mg} / \mathrm{kg}$ outside of a clinical trial setting. 


\section{Patients and methods}

\section{Patients}

This was a retrospective analysis of patients whose physician requested compassionate use of ipilimumab through the EAP. Patients with a diagnosis of unresectable stage 3/4 cutaneous, ocular or mucosal melanoma were eligible for inclusion if they were aged 16 or over, had an Eastern Cooperative Oncology Group (ECOG) PS of 0 to 2, had previously failed or were intolerant to at least one systemic therapy for metastatic melanoma and were not eligible for a clinical trial of ipilimumab. Patients with asymptomatic brain metastases at baseline were also eligible for inclusion. Previous systemic therapy should have been completed 28 days prior to treatment with ipilimumab. However, palliative radiation therapy outside of the brain, therapeutic radiation to the brain, or systemic steroids for the management of brain metastases could be administered in the 28 days prior to treatment, at the lowest fixed dose possible.

\section{Study design and treatment plan}

Patients were treated with ipilimumab at a dose of $3 \mathrm{mg} / \mathrm{kg}$, administered once every 3 weeks for four treatments. Ipilimumab was administered via a 90-minute intravenous infusion on weeks $1,4,7$ and 10. In the absence of doselimiting toxicities, it was recommended that patients receive all four doses of ipilimumab, providing their PS remained stable. Dose omission or discontinuation of treatment was recommended when necessary, based on specific safety criteria, but dose reductions or modifications of ipilimumab were not allowed. Patients who progressed after either $\geq 3$ months' stable disease (SD) or an initial objective response [complete response (CR) or partial response $(\mathrm{PR})]$ were eligible for retreatment with ipilimumab at $3 \mathrm{mg} / \mathrm{kg}$ every 3 weeks for four doses.

\section{Assessments}

Tumor assessments were performed at baseline and after completion of treatment (week 12) according to immunerelated (ir) response criteria: irCR, complete disappearance of all lesions whether measurable or not, and no new lesions; irPR, decrease in tumor burden $\geq 50 \%$ relative to baseline; irSD, not meeting criteria for irCR or irPR, in absence of ir progressive disease (irPD); irPD, increase in tumor burden $\geq 25 \%$ relative to nadir (the minimum recorded tumor burden) [27]. irCR, irPR and irPD require confirmation by a repeat, consecutive assessment at least 4 weeks from the date of first documentation [27]. Clinical benefit was defined as irCR, irPR or irSD for at least 3 months.

All patients who received ipilimumab in the EAP were monitored and assessed for AEs, including irAEs, which were graded using the Common Terminology Criteria for Adverse Events, version 3.0. AEs were managed using protocol-specific guidelines.

\section{Statistical analysis}

Patient and disease characteristics were analyzed using descriptive statistics, and expressed as relative frequencies (percentages) for discrete variables and median for continuous variables. Progression-free survival (PFS) and OS were estimated using Kaplan-Meier analysis and expressed as median values with corresponding two-sided $95 \%$ confidence intervals (CIs).

A univariate analysis and Cox proportional hazards regression model were used to analyse the relationship between prognostic factors and OS.

\section{Results}

Patients and treatment

Of 855 patients with advanced melanoma treated in the EAP at participating Italian centers, 146 had asymptomatic brain metastases at baseline. Baseline patient characteristics are provided in Table 1. As per protocol, all patients had received systemic therapy before treatment with ipilimumab, including 20 patients (14\%) who had received three or more prior therapies. Twenty-two patients $(15 \%)$ had been treated with a BRAF inhibitor, all of whom had brain metastases at the time of BRAF inhibition. Of these 22 patients, $3(18 \%)$ had a CR to BRAF-inhibitor treatment, $6(35 \%)$ had a PR and 5 (29\%) had SD, for a DCR of $82 \%$. Among 26 patients (18\%) who were being treated with steroids at enrollment, $13(9 \%)$ continued steroid treatment during ipilimumab therapy. Six patients $(4 \%)$ had received prior radiotherapy for brain metastasis.

Among the 146 treated patients, $74(51 \%)$ received all four doses of ipilimumab, 28 (19\%) received three doses, $18(12 \%)$ received two doses and $26(18 \%)$ received one dose. One patient $(1 \%)$ with an irPR and $2(1 \%)$ with irSD as their best response to induction therapy were retreated with ipilimumab at a dose of $3 \mathrm{mg} / \mathrm{kg}$ upon disease progression; neither regained disease control. The most common reasons for not completing treatment were early progression $(n=27)$, death $(n=15)$, study drug toxicity $(n=7)$ or unrelated AEs $(n=3)$. 
Efficacy

Of the 146 patients, 145 were evaluable for response (1 patient was lost to follow-up). Four patients (3\%) achieved an irCR and $13(9 \%)$ an irPR, for a global immune-related best overall response rate of $11 \%$ (Table 2). Median time to onset of response was 3.4 months (range 3.0-9.7 months) for patients with an irCR, and 3.0 months (range 2.3-6.2 months) for patients with an irPR. Twenty-two (15\%) patients achieved irSD, giving a global irDCR of $27 \%$. The median duration of objective response (irCR or irPR) was 9.7 months (range 4.0-17.0 months), while median duration of irSD was 9.4 months (range 2.9-29.3 months). Of the 26 patients receiving steroid therapy at baseline, 2 (8\%) achieved an irPR and $2(8 \%)$ achieved irSD, for a global irDCR of $15 \%$. Of the remaining 119 patients, 4 (3\%) achieved an irCR, $11(9 \%)$ an irPR and $20(16 \%)$ irSD, for a global irDCR of $29 \%$.

With a median follow-up of 20 months (range 1 to $29+$ months), estimated using Kaplan-Meier reverse

Table 1 Baseline patient characteristics

\begin{tabular}{ll}
\hline Characteristic & $n=146$ \\
\hline Median age, years (range) & $54(17-78)$ \\
Sex (female/male) $[n(\%)]$ & $70(48) / 76(52)$ \\
ECOG PS $[n(\%)]$ & \\
0 & $85(58)$ \\
1 & $57(39)$ \\
2 & $4(3)$ \\
Time from diagnosis, months (range) & $39(4-260)$ \\
Patients with liver metastases $[n(\%)]$ & $55(38)$ \\
Patients with ocular melanoma $[n(\%)]$ & $1(1)$ \\
Patients with mucosal melanoma $[n(\%)]$ & $5(3)$ \\
Elevated LDH (>480 IU/L) $[n(\%)]^{\mathrm{a}}$ & $52(45)$ \\
Number of previous therapies & \\
1 & $79(54)$ \\
2 & $47(32)$ \\
$\geq 3$ & $20(14)$ \\
Previous therapy $[n(\%)]$ & \\
Dacarbazine & $52(36)$ \\
Fotemustine & $59(40)$ \\
Temozolomide & $79(54)$ \\
Platinum-based chemotherapy & $54(37)$ \\
Interferon & $20(14)$ \\
BRAF inhibitor & $22(15)$ \\
Received prior radiotherapy for brain metastasis & $6(4)$ \\
{$[n(\%)]$} & \\
\hline & \\
\hline
\end{tabular}

ECOG Eastern Cooperative Oncology Group, $L D H$ lactate dehydrogenase, $P S$ performance status

${ }^{a}$ Out of 115 patients evaluated for LDH levels methodology, median OS was 4.3 months $(95 \% \mathrm{CI}$ 3.4-5.2) (Fig. 1). The 1-year OS rate was $20 \%(95 \% \mathrm{CI}$ 13-26). Median PFS was 3.1 months (95\% CI 2.7-3.5), and the 1-year PFS rate was $17 \%$ (95\% CI 10-23) (Fig. 1). Median OS among the six patients who had received prior radiotherapy for brain metastases was 5.5 months (range 2.2-6.7 months); however the number of patients was too small to perform any statistical analyses. Median OS among 20 patients previously receiving interferon therapy was 4.1 months (similar to that of the overall population of patients with brain metastases).

Univariate analysis showed that younger age (age $<60$ years; median OS: 3.7 months), ECOG PS of 2 (median OS: 3.2 months), presence of liver metastases (median OS: 3.7 months), LDH > 480 IU/L (median OS: 3.4 months), and steroid use at baseline (median OS: 2.9 months) were significantly associated with poorer survival (Table 3 ). After using a Cox proportional hazards model to adjust for prognostic variables, use of steroids, age and ECOG PS were significantly associated with OS.

Safety and tolerability

58 patients (40\%) with asymptomatic brain metastases reported an $\mathrm{AE}$ of any grade. These were considered treatment-related in 42 patients (29\%) (Table 4). Grade III/IV AEs were reported in 15 patients $(10 \%)$ and were considered treatment-related in nine patients $(6 \%)$. The most common grade III/IV AEs were liver dysfunction, which occurred in four patients $(3 \%)$ and diarrhea, which occurred in two patients $(6 \%)$. The patients with liver dysfunction did not have liver metastases. AEs were generally reversible with treatment as per protocol-specific guidelines, and the median time to resolution of treatmentrelated AEs was 1.6 weeks (range 0.1-3.4). CNS events such as hemorrhage, headache and seizure were reported in ten patients $(6.9 \%)$, and were grade III/IV in five patients $(3.4 \%)$. Grade III headache and grade III confusion were

Table 2 Tumor response

\begin{tabular}{lc}
\hline Response according to irRC & Patients $[n(\%)]$ \\
\hline irCR & $4(3)$ \\
irPR & $13(9)$ \\
irSD & $22(15)$ \\
Immune-related progressive disease & $106(73)$ \\
Immune-related best overall response rate & $17(12)$ \\
irDCR & $39(27)$ \\
\hline
\end{tabular}

irCR immune-related complete response, $\operatorname{irDCR}$ immune-related disease control rate, $\operatorname{ir} P R$ immune-related partial response, irRC immune-related response criteria, ir $S D$ immune-related stable disease 
Fig. 1 Kaplan-Meier curves of OS and PFS in patients with metastatic melanoma and brain metastases. $C I$ confidence interval, $O S$ overall survival

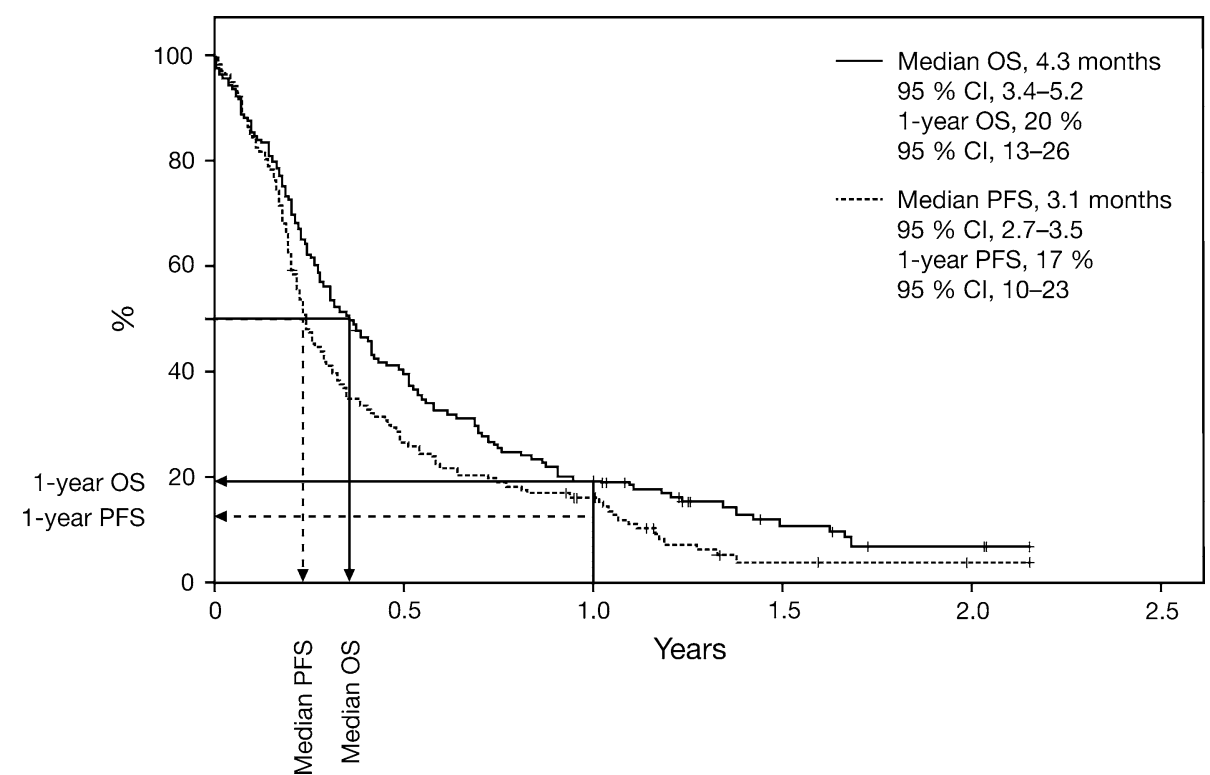

Table 3 Univariate analysis of survival by baseline characteristic

\begin{tabular}{lll}
\hline Characteristic at baseline & $\begin{array}{l}\text { Median OS } \\
\text { (months) }\end{array}$ & $p$ \\
\hline Age, years $(n)$ & & 0.06 \\
$\quad<60$ (91) & 3.7 & \\
$\geq 60$ (55) & 5.5 & $<0.0001$ \\
ECOG PS (n) & & \\
0 (85) & 6.0 & \\
1-2 (61) & 3.2 & \\
Presence of liver metastases (n) & & \\
$\quad$ Yes (55) & 3.7 & 0.06 \\
No (91) & 4.5 & \\
LDH (n) & & \\
$>480$ IU/L (52) & 3.4 & \\
$\leq 480$ IU/L (63) & 6.2 & 0.05 \\
Previous use of BRAF inhibitor & & \\
$\quad(n)$ & & \\
Yes (22) & 2.5 & \\
No (124) & 5.0 & \\
Steroid use (n) & & \\
Yes (26) & 2.9 & \\
No (120) & 4.9 & \\
& &
\end{tabular}

ECOG Eastern Cooperative Oncology Group, $L D H$ lactate dehydrogenase, $O S$ overall survival, $P S$ performance status

each reported in 1 patient, and were considered to be possibly related to treatment (Table 4).

Of the 17 patients with an irCR or irPR, three patients had grade I/II AEs that were considered related to ipilimumab treatment. None had grade III/IV treatment-related AEs.
Table 4 Treatment-related AEs

\begin{tabular}{lcl}
\hline Treatment-related AE & \multicolumn{2}{l}{ Patients $[n(\%)]$} \\
\cline { 2 - 3 } & Any grade & Grade 3/4 \\
\hline Total & $42(29)$ & $9(6)$ \\
Diarrhea & $14(10)$ & $2(1)$ \\
Nausea & $7(5)$ & $1(1)$ \\
Vomiting & $5(3)$ & $1(1)$ \\
Asthenia & $10(7)$ & $1(1)$ \\
Pruritus & $4(3)$ & 0 \\
Rash & $4(3)$ & 0 \\
Liver toxicity & $4(3)$ & $4(3)$ \\
Fever & $3(2)$ & 0 \\
Headache & $1(1)$ & $1(1)$ \\
Confusion & $1(1)$ & $1(1)$
\end{tabular}

$A E$ adverse event

\section{Discussion}

The EAP allowed the efficacy and safety of ipilimumab to be evaluated in a large cohort of patients with melanoma and brain metastases in a setting that closely reflected daily clinical practice. Median OS for patients with melanoma and asymptomatic brain metastases was 4.3 months and approximately $20 \%$ of patients were alive at 1 year. A similar analysis of data from an EAP conducted in the United States showed that among 715 patients with melanoma brain metastases, $25 \%$ were alive 1 year after starting treatment with ipilimumab $3 \mathrm{mg} / \mathrm{kg}$ [28]. Because it can take time to build an immune response against the tumor, median OS does not necessarily reflect the potential of ipilimumab to provide durable clinical benefit. It is 
therefore important to consider those patients surviving beyond the median $[11,27]$. Ipilimumab activity has been shown to persist in the absence of continued treatment, with survival curves plateauing after 2-3 years and a significant proportion of patients surviving 4-5 years after initiation of treatment $[15,16,29]$. Consequently, it is possible that ipilimumab may provide long-term survival in a significant proportion of patients with brain metastases, despite the modest median OS reported in this EAP. This will be confirmed with longer follow-up.

The irDCR in this analysis was $27 \%$, which is consistent with DCRs reported in clinical trials of ipilimumab. For example, in the registrational phase 3 trial of patients with pretreated metastatic melanoma, the DCR according to modified World Health Organization criteria was $29 \%$ for the 137 patients receiving ipilimumab $3 \mathrm{mg} / \mathrm{kg}$ as monotherapy. This included 15 patients $(11 \%)$ who had stable brain metastases at baseline [13].

Of note, patients who were receiving steroid therapy at baseline in the EAP tended to have a poorer irDCR than those who were not (15 vs. $29 \%$ ), and had a significantly poorer median OS. Similar results have been reported in patients treated with ipilimumab $10 \mathrm{mg} / \mathrm{kg}$ in a phase 2 trial, as previously discussed [19]. This variation in outcome may simply reflect the fact that patients requiring steroid therapy have features associated with a poorer prognosis, such as higher burden of brain disease and neurological symptoms. However, it is also possible that steroids have an independent, negative effect on ipilimumab activity. Although studies have suggested that systemic steroids do not interfere with ipilimumab efficacy when used to manage treatment-related AEs [30, 31], the effect of steroid use prior to treatment with ipilimumab is unclear. Indeed, in a preclinical model, glucocorticoids were shown to inhibit naïve T-cells, while not impairing the antitumor activity of activated T-cells [32].

In this analysis, median OS was reduced for the 22 patients who had previously been treated with a BRAF inhibitor, and univariate analysis revealed a trend for prior BRAF use to correlate with shortened survival despite ipilimumab treatment. Interestingly, a previous analysis of patients treated sequentially with ipilimumab and a BRAF inhibitor, or vice versa, showed that patients who received ipilimumab upon disease progression with a BRAF inhibitor had significantly shorter survival [33]. This may be because patients were unable to complete subsequent treatment with ipilimumab due to rapid disease progression $[33,34]$.

The use of ipilimumab in patients with brain metastases could cause neurological complications due to inflammation in the brain [19]. However, the results of previous studies suggest that the presence of brain metastases has no effect on the type and frequency of AEs experienced with ipilimumab treatment $[19,20]$. In the EAP, the percentage of treatment-related AEs of any grade was lower for patients with brain metastases than for the total population [35], and AEs were generally manageable. CNS AEs were experienced by 10 patients ( $6.9 \%$ ), including hemorrhage, headache, and seizure, but were only considered treatmentrelated in two patients $(1 \%)$.

Because of its retrospective nature, this subanalysis is subject to limitations. For example, brain scans were not required for enrollment; therefore, data may not be fully representative of the EAP population. Furthermore, data on intracranial versus extracranial progression and/or response rates were not available. The results therefore require validation in prospective clinical trials. However, the data presented here, together with previously published data, suggest that ipilimumab provides durable clinical benefit and has a manageable side-effect profile in patients with advanced melanoma and brain metastases. Further prospective evaluation is warranted in this hard-to-treat patient population.

Acknowledgments This work was supported in part by the Associazione Italiana per la Ricerca sul Cancro, the Italian Ministry of Health, via the Ricerca Finalizzata 2010. The Expanded Access Program (EAP) was sponsored by Bristol-Myers Squibb (BMS). Editorial and writing assistance was provided by StemScientific, funded by BMS. Statistical support was provided by a clinical research organization, Clinical Research Services, and was funded by BMS. The authors would like to thank the patients and investigators who participated in the European EAP.

Conflict of interest Paola Queirolo has received honoraria from and served as a consultant and in an advisory role for BMS, GlaxoSmithKline (GSK) and Roche-Genentech. Paolo Ascierto has served as a consultant or in an advisory role for BMS, Merck Sharp and Dohme, Roche-Genentech, GSK, Amgen, Celgene and Novartis; he has received honoraria from BMS, Merck Sharp \& Dohme and Roche-Genentech, and research funding from BMS. Paolo Marchetti has received honoraria for seminar/congress presentations from BMS, GSK, Pfizer, Roche-Genentech, AstraZeneca, Sanofi Aventis, Novartis, Astellas and Ipsen. Michele Del Vecchio has served as a consultant or in an advisory role for Merck/Schering-Plough, and GSK; he has received research funding from Celgene, Novartis and Roche. Michele Maio has had an advisory role and received funding for communication programs from BMS, Roche, and MSD and has received research funding from BMS. Vanna Chiarion Sileni had a consulting and advisory role for BMS, MSD, Roche-Genentech, and GSK. All other authors confirm there are no financial conflicts of interest to disclose.

Ethical standards The EAP protocol was approved by the local independent ethics committees and all participating patients provided signed informed consent before enrollment.

\section{References}

1. Fife KM, Colman MH, Stevens GN, Firth IC, Moon D, Shannon KF, Harman R, Petersen-Schaefer K, Zacest AC, Besser M, Milton GW, McCarthy WH, Thompson JF (2004) Determinants of outcome in melanoma patients with cerebral metastases. J Clin Oncol 22:1293-1300 
2. Sloan AE, Nock CJ, Einstein DB (2009) Diagnosis and treatment of melanoma brain metastasis: a literature review. Cancer Control $16: 248-255$

3. Majer M, Jensen RL, Shrieve DC, Watson GA, Wang M, Leachman SA, Boucher KM, Samlowski WE (2007) Biochemotherapy of metastatic melanoma in patients with or without recently diagnosed brain metastases. Cancer 110:1329-1337

4. Staudt M, Lasithiotakis K, Leiter U, Meier F, Eigentler T, Bamberg M, Tatagiba M, Brossart P, Garbe C (2010) Determinants of survival in patients with brain metastases from cutaneous melanoma. Br J Cancer 102:1213-1218. doi:10.1038/sj.bjc.6605622

5. Bhangoo SS, Linskey ME, Kalkanis SN, American Association of Neurologic Surgeons (AANS), Congress of Neurologic Surgeons (CNS) (2011) Evidence-based guidelines for the management of brain metastases. Neurosurg Clin N Am 22:97-104, viii. doi:10.1016/j.nec.2010.09.001

6. McWilliams RR, Brown PD, Buckner JC, Link MJ, Markovic SN (2003) Treatment of brain metastases from melanoma. Mayo Clin Proc 78:1529-1536

7. Gerstner ER, Fine RL (2007) Increased permeability of the blood-brain barrier to chemotherapy in metastatic brain tumors: establishing a treatment paradigm. J Clin Oncol 25:2306-2312

8. Yonemori K, Tsuta K, Ono M, Shimizu C, Hirakawa A, Hasegawa T, Hatanaka Y, Narita Y, Shibui S, Fujiwara Y (2010) Disruption of the blood brain barrier by brain metastases of triplenegative and basal-type breast cancer but not HER2/neu-positive breast cancer. Cancer 116:302-308. doi:10.1002/cncr.24735

9. Dummer R, Goldinger S, Turtschi C, Eggmann N, Michielin O, Mitchell L, Veronese L, Hilfiker PR, Rinderknecht JD (2012) Open-label pilot study of vemurafenib in previously treated metastatic melanoma patients with symptomatic brain metastases. Ann Oncol 23(9 Suppl):1125P

10. Long GV, Trefzer U, Davies MA, Kefford RF, Ascierto PA, Chapman PB, Puzanov I, Hauschild A, Robert C, Algazi A, Mortier L, Tawbi H, Wilhelm T, Zimmer L, Switzky J, Swann S, Martin AM, Guckert M, Goodman V, Streit M, Kirkwood JM, Schadendorf D (2012) Dabrafenib in patients with Val600Glu or Val600Lys BRAF-mutant melanoma metastatic to the brain (BREAK-MB): a multicentre, open-label, phase 2 trial. Lancet Oncol 13:1087-1095. doi:10.1016/S1470-2045(12)70431-X

11. Hoos A, Ibrahim R, Korman A, Abdallah K, Berman D, Shahabi V, Chin K, Canetta R, Humphrey R (2010) Development of ipilimumab: contribution to a new paradigm for cancer immunotherapy. Semin Oncol 37:533-546. doi:10.1053/j.seminoncol. 2010.09.015

12. YERVOY ${ }^{\mathrm{TM}}$ Summary of Product Characteristics. http://www. medicines.org.uk/emc/medicine/24779/SPC/yervoy $\% 205 \%$ $20 \mathrm{mg} \sim \mathrm{ml} \% 20$ concentrate $\% 20$ for $\% 20$ solution $\% 20$ for $\% 20 \mathrm{infu}$ sion/\#INDICATIONS. Accessed on 6 Jan 2014

13. Hodi FS, O'Day SJ, McDermott DF, Weber RW, Sosman JA, Haanen JB, Gonzalez R, Robert C, Schadendorf D, Hassel JC, Akerley W, van den Eertwegh AJ, Lutzky J, Lorigan P, Vaubel JM, Linette GP, Hogg D, Ottensmeier CH, Lebbé C, Peschel C, Quirt I, Clark JI, Wolchok JD, Weber JS, Tian J, Yellin MJ, Nichol GM, Hoos A, Urba WJ (2010) Improved survival with ipilimumab in patients with metastatic melanoma. N Engl J Med 363:711-723. doi:10.1056/NEJMoa1003466

14. Robert C, Thomas L, Bondarenko I, O’Day S, Weber J, Garbe C, Lebbe C, Baurain JF, Testori A, Grob JJ, Davidson N, Richards J, Maio M, Hauschild A, Miller WH Jr, Gascon P, Lotem M, Harmankaya K, Ibrahim R, Francis S, Chen TT, Humphrey R, Hoos A, Wolchok JD (2011) Ipilimumab plus dacarbazine for previously untreated metastatic melanoma. $\mathrm{N}$ Engl $\mathrm{J}$ Med 364:2517-2526. doi:10.1056/NEJMoa1104621

15. Lebbe C, Weber JS, Maio M, Neyns B, Harmankaya K, Chin K, Opatt McDowell D, Cykowski L, McHenry MB, Wolchok JD
(2012) Five-year survival rates for patients with metastatic melanoma treated with ipilimumab in phase II trials. Ann Oncol 23(9 Suppl):1116PD

16. Prieto PA, Yang JC, Sherry RM, Hughes MS, Kammula US, White DE, Levy CL, Rosenberg SA, Phan GQ (2012) CTLA-4 blockade with ipilimumab: long-term follow-up of 177 patients with metastatic melanoma. Clin Cancer Res 18:2039-2047. doi:10.1158/1078-0432.CCR-11-1823

17. Ibrahim RA, Berman DM, DePril V, Humphrey RW, Chen T, Messina M, Chin KM, Liu HY, Bielefield M, Hoos A (2011) Ipilimumab safety profile: summary of findings from completed trials in advanced melanoma. J Clin Oncol 29(15 Suppl):8583

18. Weber J (2007) Review: anti-CTLA-4 antibody ipilimumab: case studies of clinical response and immune-related adverse events. Oncologist 12:864-872

19. Margolin K, Ernstoff MS, Hamid O, Lawrence D, McDermott D, Puzanov I, Wolchok JD, Clark JI, Sznol M, Logan TF, Richards J, Michener T, Balogh A, Heller KN, Hodi FS (2012) Ipilimumab in patients with melanoma and brain metastases: an open-label, phase 2 trial. Lancet Oncol 13:459-465. doi:10.1016/S14702045(12)70090-6

20. Di Giacomo AM, Ascierto PA, Pilla L, Santinami M, Ferrucci PF, Giannarelli D, Marasco A, Rivoltini L, Simeone E, Nicoletti SV, Fonsatti E, Annesi D, Queirolo P, Testori A, Ridolfi R, Parmiani G, Maio M (2012) Ipilimumab and fotemustine in patients with advanced melanoma (NIBIT-M1): an open-label, single-arm phase 2 trial. Lancet Oncol 13:879-886. doi:10.1016/ S1470-2045(12)70324-8

21. Weber JS, Amin A, Minor D, Siegel J, Berman D, O’Day SJ (2011) Safety and clinical activity of ipilimumab in melanoma patients with brain metastases: retrospective analysis of data from a phase 2 trial. Melanoma Res 21:530-534. doi:10.1097/CMR. 0b013e $32834 \mathrm{~d} 3 \mathrm{~d} 88$

22. Lebbe C, McDermott DF, Robert C, Lorigan P, Ottensmeier CH, Wolchok J, Garbe C, Messina M, Hoos A, Weber JS (2010) Ipilimumab improves survival in previously treated, advanced melanoma patients with poor prognostic factors: subgroup analyses from a phase III trial. Ann Oncol 21(8 Suppl):13240

23. Heller K, Pavlick AC, Hodi FS, Thompson JA, Margolin KA, Lawrence DP, McDermott DF, Samlowski WE, Michener T, Karasarides M (2011) Safety and survival analysis of ipilimumab therapy in patients with stable asymptomatic brain metastases. J Clin Oncol 29(15 Suppl):8581

24. Engelhardt B, Coisne C (2011) Fluids and barriers of the CNS establish immune privilege by confining immune surveillance to a two-walled castle moat surrounding the CNS castle. Fluids Barriers CNS 8:4. doi:10.1186/2045-8118-8-4

25. Wilson EH, Weninger W, Hunter CA (2010) Trafficking of immune cells in the central nervous system. J Clin Invest 120:1368-1379. doi:10.1172/JCI41911

26. Queirolo P, Simeone E, De Galitiis F, Di Guardo L, Di Giacomo AM, Marconcini R, Ferraresi V, De Rosa F, Guida M, Stragliotto S (2012) Efficacy and safety data from patients with advanced melanoma and brain metastases participating in the European ipilimumab Expanded Access Programme in Italy. Ann Oncol 23(9 Suppl):1132P

27. Wolchok JD, Hoos A, O'Day S, Weber JS, Hamid O, Lebbé C, Maio M, Binder M, Bohnsack O, Nichol G, Humphrey R, Hodi FS (2009) Guidelines for the evaluation of immune therapy activity in solid tumors: immune-related response criteria. Clin Cancer Res 15:7412-7420. doi:10.1158/1078-0432.CCR-091624

28. Lawrence D, McDermott D, Hamid O, Weber JS, Wolchok J, Richards J, Minor D, Pavlick A, Sznol M, Hwu P, Urba W, Amin A, Bennett K, Michener T, Balogh A, Hodi F (2012) Treatment of patients with stage III or IV melanoma on an ipilimumab 
Expanded Access Program: results for $3 \mathrm{mg} / \mathrm{kg}$ cohort. Pigment Cell Melanoma Res 25(6):868

29. Maio M, Bondarenko I, Robert C, Thomas L, Garbe C, Testori A, Francis S, Chin K, Wolchok J (2012) Four-year survival update for metastatic melanoma patients treated with ipilimumab plus dacarbazine in phase 3 study CA184-024. Ann Oncol 23(9 Suppl):1127P

30. Weber J, Thompson JA, Hamid O, Minor D, Amin A, Ron I, Ridolfi R, Assi H, Maraveyas A, Berman D, Siegel J, O’Day SJ (2009) A randomized, double-blind, placebo-controlled, phase II study comparing the tolerability and efficacy of ipilimumab administered with or without prophylactic budesonide in patients with unresectable stage III or IV melanoma. Clin Cancer Res 15:5591-5598. doi:10.1158/1078-0432.CCR-09-1024

31. Tarhini A, Lo E, Minor DR (2010) Releasing the brake on the immune system: ipilimumab in melanoma and other tumors. Cancer Biother Radiopharm 25:601-613. doi:10.1089/cbr 2010.0865

32. Hinrichs CS, Palmer DC, Rosenberg SA, Restifo NP (2005) Glucocorticoids do not inhibit antitumor activity of activated CD8 + T cells. J Immunother 28:517-524
33. Ackerman A, McDermott DF, Lawrence DP, Gunturi A, Flaherty KT, Giobbie-Hurder A, Hodi FS, Ibrahim N, Atkins MB, Cho DC, Sullivan RJ (2012) Outcomes of patients with malignant melanoma treated with immunotherapy prior to or after vemurafenib. J Clin Oncol 30(15 Suppl):8569

34. Ascierto PA, Simeone E, Giannarelli D, Grimaldi AM, Romano A, Mozzillo N (2012) Sequencing of BRAF inhibitors and ipilimumab in patients with metastatic melanoma: a possible algorithm for clinical use. J Transl Med 10:107. doi:10.1186/14795876-10-107

35. Ascierto PA, Chiarion Sileni V, Del Vecchio M, Altomonte M, De Galitiis F, Ridolfi L, Cognetti F, Testori A, Bernengo MG, Queirolo P (2012) The European ipilimumab Expanded Access Programme: efficacy and safety data from the Italian cohort of patients with pretreated, advanced melanoma. Ann Oncol 23(9 Suppl):1128P 